\title{
Risk factors for coronary artery lesions in children with Kawasaki disease
}

\author{
Junyan Duan, BM ${ }^{a}$, Huanhuan Jiang, $B M^{a}$ and Ming $L u, M M^{b}$
}

People's Hospital Jiangsu Province, P. R. China.

b. Department of Pediatrics, Affiliated Hospital of Xuzhou Medical University, Xuzhou 221000, Jiangsu Province, P. R. China.

E-mail address:

Huanhuan Jiang, BM:

bridgettruizscu@aol.com

Funding:

None.

Conflict of interest:

None.

Received: 10-24-2019

Accepted: 5-22-2020 To cite: Duan J, Jiang H, Lu M. Risk factors for

\begin{abstract}
Introduction. Kawasaki disease (KD) is a nonspecificsystemic vasculitic disease that frequently occurs among children, and coronary artery lesion (CAL) is the most serious complication. Objectives. We aimed to study the risk factors for CAL in children with KD.

Materials and methods. KD children in accordance with diagnostic criteria, who were hospitalized from January 2014 to December 2017, were selected as subjects. Univariate and multivariate logistic regression analyses were conducted to explore the relationships between CAL and gender, age, clinical diagnosis, erythrocyte sedimentation rate (ESR), platelet count, hemoglobin level, Creactive protein level, white blood cell count, initiation time of IVIG administration and duration of fever.

Results. The enrolled 982 KD children were divided into a CAL group $(\mathrm{n}=104)$ and an NCAL group $(n=878)$ according to cardiac color Doppler ultrasonography. The incidence rate of CAL was $10.6 \%$ (104/982). Univariate analysis showed that the two groups had significantly different gender, ESR, platelet count, initiation time of IVIG administration and duration of fever $(\mathrm{P}<0.05)$. Multivariate logistic regression analysis revealed that male gender, elevated ESR and delayed use of IVIG were independent risk factors for KD complicated with CAL.

Conclusions: Male gender, increased ESR and delayed use of IVIG were independent risk factors for KD complicated with CAL.

Key words: Kawasaki disease, coronary artery, logistic regression, risk factors.
\end{abstract}

http:/ / dx.doi.org/10.5546/ aap.2020.eng.327

\section{INTRODUCTION}

Kawasaki disease (KD) is a nonspecific systemic vasculitic disease that frequently occurs among children, and coronary artery lesion (CAL) is the most serious complication, with an incidence rate of $20 \% \sim 35 \%$. ${ }^{1}$ Accumulating evidence has proven that KD-induced CAL persists for many years, and may cause coronary heart disease in adulthood. ${ }^{2-4} \mathrm{CAL}$ has replaced rheumatic fever to be one of the main causes for acquired heart disease among children. In recent years, the early diagnosis and treatment of KD are still difficult due to unknown etiology. ${ }^{5} \mathrm{KD}$ can lead to CAL which may induce thrombotic infarction and stenosis, myocardial infarction and even sudden death. Therefore, it is imperative to find the risk factors as soon as possible and to take reasonable and effective measures in time to reduce the incidence and mortality rates of KD. In this study, the objective was to explore the risk factors for CAL in children with KD.

\section{MATERIALS AND METHODS}

A single-center large-sample study coronary artery lesions in children with Kawasaki disease. Arch Argent Pediatr 2020;118(5):327-331. was performed to analyze the clinical data of children with KD.

\section{Baseline clinical data}

KD children who were hospitalized from January 2014 to December 2017 were selected. This study has been approved by the ethics committee of our hospital on January 4th, 2014 (Approval No. AHXMU 2014-01002), and signed informed consent has been obtained from the guardians of all participants.

\section{Diagnostic criteria}

KD was diagnosed according to 
the guidelines for the diagnosis and treatment of KD proposed by the American Academy of Pediatrics and the American Heart Association in $2004 .{ }^{6} \mathrm{~A}$ child who had a fever $\left(39-40^{\circ} \mathrm{C}\right)$ duration of $>5 \mathrm{~d}$ and 4 of the following 5 clinical manifestations was diagnosed as KD: 1) changes in the four limbs: Palmoplantar erythema in the acute phase, hard edema of hands and feet, finger (toe) tip membranous peeling during recovery; 2) pleomorphic rash; 3) bilateral conjunctival hyperemia; 4) congestion and cracking of lips, diffuse congestion of oral mucosa, and protruding papillae (strawberry tongue); 5) enlargement of cervical lymph nodes. If a child had a fever (39$40^{\circ} \mathrm{C}$ ) duration of $>5 \mathrm{~d}$, without meeting 4 of the above 5 clinical manifestations, but cardiac color Doppler ultrasonography disclosed coronary dilatation or febrile diseases such as scarlet fever, he/she was diagnosed as incomplete KD after drug hypersensitivity syndrome, Stevens-Johnson syndrome, toxic shock syndrome, adenovirus infection or Epstein-Barr virus infection were excluded.

According to the formula of the normal value of the coronary artery corrected by body surface area developed by de Zorzi et al., ${ }^{7} \mathrm{Z}$ was calculated by: $Z=$ (Observation value - mean normal value)/standard deviation. CAL was defined when the inner diameter $Z$ value of the left anterior descending coronary artery, or left main or right coronary artery exceeded 2.5. Coronary artery dilation was defined when $\mathrm{Z}$ was $>2.5$ and the inner diameter of the coronary artery was $\geq 4 \mathrm{~mm}$. Coronary aneurysm: Small coronary aneurysm (inner diameter of coronary artery: $>4 \mathrm{~mm}$ and $<5 \mathrm{~mm}$ ), middle coronary aneurysm (inner diameter: $5-8 \mathrm{~mm}$ ) and giant coronary aneurysm (inner diameter: $>8 \mathrm{~mm}$ ). ${ }^{8}$ Body surface area was calculated as follows. ${ }^{9}$ When body weight was $<30 \mathrm{~kg}$, body surface area $\left(\mathrm{m}^{2}\right)=$ body mass $(\mathrm{kg}) \times 0.035\left(\mathrm{~m}^{2} / \mathrm{kg}\right)+0.1\left(\mathrm{~m}^{2}\right)$; when body weight was $30-50 \mathrm{~kg}$, the body surface area was elevated by $0.1 \mathrm{~m}^{2}$ for every $5 \mathrm{~kg}$ weight increase.

\section{Statistical analysis}

All data were statistically analyzed by SPSS 17.0 software. Intergroup comparisons for numerical data were performed by the $\chi^{2}$ test, and the quantitative data conforming to normal distribution were expressed as mean \pm standard deviation $(x \pm S D)$. Univariate and multivariate logistic regression analyses were carried out to clarify the relationships of gender, age, clinical diagnosis, erythrocyte sedimentation rate (ESR), platelet count, hemoglobin level, $C$ reactive protein (CRP) level, white blood cell count, initiation time of IVIG administration and duration of fever with CAL. Univariate analysis was conducted with the independent sample $t$ test, and the variables with statistical significance were subjected to multivariate logistic regression analysis. $\mathrm{P}<0.05$ was considered statistically significant.

TABLE 1. Univariate analysis of risk factors for CAL in children with KD

\begin{tabular}{|c|c|c|c|c|}
\hline & CAL group $(n=104)$ & NCAL group $(n=878)$ & $t / \chi^{2}$ & $p$ \\
\hline \multicolumn{5}{|l|}{ Gender } \\
\hline Male & 80 & 518 & 12.547 & 0.000 \\
\hline Female & 24 & 360 & & \\
\hline Age (month) & $30.47 \pm 5.48$ & $31.38 \pm 5.78$ & 1.526 & 0.127 \\
\hline \multicolumn{5}{|l|}{ Clinical diagnosis } \\
\hline Typical KD & 60 & 576 & 2.550 & 0.110 \\
\hline Incomplete KD & 44 & 302 & & \\
\hline $\operatorname{ESR}(\mathrm{mm} / \mathrm{h})$ & $69.62 \pm 9.18$ & $82.71 \pm 8.94$ & 14.079 & 0.000 \\
\hline Platelet count $\left(\times 10^{9} / \mathrm{L}\right)$ & $352.68 \pm 23.48$ & $432.58 \pm 24.17$ & 31.972 & 0.000 \\
\hline Hemoglobin level (g/L) & $9.36 \pm 1.95$ & $9.67 \pm 1.54$ & 1.882 & 0.060 \\
\hline $\mathrm{CRP}(\mathrm{mg} / \mathrm{L})$ & $69.23 \pm 6.15$ & $68.89 \pm 6.35$ & 0.518 & 0.605 \\
\hline White blood cell count $\left(\times 10^{9} / \mathrm{L}\right)$ & $15.46 \pm 2.11$ & $15.24 \pm 2.32$ & 0.923 & 0.356 \\
\hline Initiation time of IVIG use (d) & $6.7 \pm 1.5$ & $9.5 \pm 1.6$ & 16.984 & 0.000 \\
\hline Duration of fever $(\mathrm{d})$ & $8.3 \pm 1.2$ & $9.7 \pm 1.3$ & 10.466 & 0.000 \\
\hline
\end{tabular}

CAL: Coronary artery lesion; KD: Kawasaki disease; ESR: erythrocyte sedimentation rate; CRP: C reactive protein. 


\section{RESULTS}

\section{Baseline clinical data}

The $982 \mathrm{KD}$ cases consisted of 598 boys and 384 girls. The onset ages of $64(6.5 \%), 814(82.9 \%)$ and $104(10.6 \%)$ cases were $\leq 6$ months, 6 months 5 years and $>5$ years, respectively. A total of 634 cases $(64.6 \%)$ had typical KD, and the other $348(35.4 \%)$ had incomplete KD. The enrolled KD children were divided into a CAL group ( $\mathrm{n}=104)$ and an NCAL group $(\mathrm{n}=878)$ according to cardiac color Doppler ultrasonography, who were all diagnosed for the first time. The incidence rate of CAL was $10.6 \%$ (104/982).

\section{Univariate analysis of risk factors for CAL in children with KD}

Univariate analysis showed that the two groups had significantly different gender, ESR, platelet count, initiation time of IVIG administration and duration of fever $(\mathrm{P}<0.05)$ (Table 1).

\section{Multivariate logistic analysis of risk factors for CAL in children with KD}

Multivariate logistic regression analysis revealed that male gender, elevated ESR and delayed use of IVIG were independent risk factors for KD complicated with CAL (Table 2).

\section{DISCUSSION}

As an immune self-limiting vasculitis involving small and medium blood vessels, ${ }^{10}$ KD often leads to coronary artery dilatation or giant aneurysm formation, and potential risks of myocardial infarction and even sudden cardiac death. ${ }^{11}$ Therefore, the early diagnosis and timely treatment of KD have attracted widespread attention, and the risk factors for KD complicated with CAL have also become the focus of pediatricians.
Age of $<1$ or $>8$ years old, male gender, ESR, CRP level, white blood cell count, platelet count, albumin level, delayed use of low-dose IVIG and recurrence of KD have been reported to be the risk factors for CAL. ${ }^{12}$ In this study, gender (male) was an independent risk factor for CAL. Also, boys with KD may have a specific genetic predisposition to CAL. Therefore, KD can be defined as an autoimmune response that has a genetic susceptibility background and is induced by external stimuli. In addition, the high risk of CAL in specific populations also suggests that genetic susceptibility plays an important role in the pathogenesis of KD. ${ }^{13}$

Herein, age, fever duration, CRP level or white blood cell count of KD children was not significantly correlated with the occurrence of CAL. Probably, $80 \%$ of children with KD have the onset from 6 months to 4 years old. ${ }^{14}$ If children are aged $<1$ year or $>5$ years, the clinical manifestations of KD are often atypical, which cannot be diagnosed by clinicians in the early stage, so the children fail to receive IVIG treatment in time, resulting in a significantly increased incidence rate of CAL. ${ }^{15} \mathrm{KD}$ induces vascular endothelial injury by activating immune cells and enhancing immune response due to unknown reasons. ${ }^{16}$ IVIG can significantly relieve inflammation and immune response, block Fc receptors on the platelet surface, and exert non-specific anti-inflammatory effects, thereby mitigating CAL. ${ }^{17,18}$ Moreover, early use $(<10 \mathrm{~d})$ of IVIG can significantly reduce the risk of CAL in $\mathrm{KD}$, which is consistent with the results of this study. ${ }^{19}$

CRP and ESR are well-recognized laboratory markers for inflammatory response, both of which are elevated in the early stage of KD, with high diagnostic values. Kawasaki first reported in 1967 that about $36 \%$ of children with KD had desynchronized ESR and CRP

TABLE 2. Multivariate logistic analysis of risk factors for CAL in children with KD

\begin{tabular}{lcccc}
\hline Factor & $\boldsymbol{\beta}$ & Wald value & OR value & 95 $\%$ CI \\
\hline Gender & -1.009 & 7.267 & 0.364 & $0.175-0.756$ \\
ESR & 0.019 & 11.243 & 1.019 & $1.004-1.345$ \\
Initiation time of IVIG use & 0.257 & 28.895 & 1.287 & $1.145-1.458$ \\
Platelet count & 0.001 & 2.798 & 1.004 & $0.982-1.155$ \\
Duration of fever & 0.004 & 0.743 & 1.043 & $0.942-1.156$ \\
\hline
\end{tabular}

CAL: Coronary artery lesion; KD: Kawasaki disease; ESR: erythrocyte sedimentation rate. 
changes..$^{20}$ Bray et al. ${ }^{21}$ also found that about $44 \%$ of children with KD had similar manifestations. CRP is an acute phase reactive protein which increases 4-6 h after inflammatory stimulation, peaks 36-50 $\mathrm{h}$ after stimulation and decreases 3-7 d after termination of stimulation, while ESR is determined by the rate of erythrocyte decline in plasma, which is affected by the size, shape and quantity of erythrocytes and the level of immunoglobulin. ESR rises slowly and returns to the baseline slower after inflammatory factors are absent. The peak of ESR lags that of CRP. Therefore, the increase of CRP and ESR in children with KD is desynchronized. Additionally, elevated ESR suggests that KD is in the advanced stage, the inflammatory response lasts for a long time, and the coronary artery has a long-term exposure to inflammatory factors, which may give aggregated vascular lesions. Thus, the increase in ESR can reflect the damage of inflammatory response to the coronary artery to some extent. ${ }^{22}$ Notably, ESR is susceptible to plasma globulin. Accordingly, ESR before IVIG use should be used as the basis to assess the severity of KD. In 2005, Gómez de Diego et al. used multislice CT coronary angiography to evaluate the coronary anatomy. ${ }^{23}$ Recently, Goh et al. reported that coronary CT angiography was an attractive alternative to invasive coronary angiography for KD children. ${ }^{24}$ Besides, Jrad et al. found that CT coronary angiography was superior in detecting fusiform coronary lesions as well as distal and posterior networks in KD. Nevertheless, transthoracic echocardiography was still the fist-line examination owing to radiating characteristics and availability. ${ }^{25}$

Herein, male gender, elevated ESR and delayed IVIG administration were independent risk factors for KD complicated with CAL. Furthermore, the number of included observational indices remained limited. Therefore, further in-depth studies are in need to find more specific predictive indices, aiming to provide a theoretical basis for the early diagnosis and treatment of KD.

\section{CONCLUSIONS}

In summary, male gender, increased ESR and delayed use of IVIG were independent risk factors for KD children complicated with CAL.

\section{REFERENCES}

1. Patel RM, ShulmanST. Kawasaki disease: a comprehensive review of treatment options. J Clin Pharm Ther. 2015; 40(6):620-5.
2. Kim MK, Song MS, Kim GB. Factors Predicting Resistance to Intravenous Immunoglobulin Treatment and Coronary Artery Lesion in Patients with Kawasaki Disease: Analysis of the Korean Nationwide Multicenter Survey from 2012 to 2014. Korean Circ J. 2018; 48(1):71-9.

3. Xie T, Wang Y, Fu S, Wang W, et al. Predictors for intravenous immunoglobulin resistance and coronary artery lesions in Kawasaki disease. Pediatr Rheumatol Online J. 2017; 15(1):17.

4. Lee HY, Song MS. Predictive factors of resistance to intravenous immunoglobulin and coronary artery lesions in Kawasaki disease. Korean J Pediatr. 2016; 59(12):477-82.

5. Li XQ, Kuang L, Wu J, Hu C, et al. [Progress in treatment of Kawasaki disease]. J Modern Clin Med. 2016; 42:328-30.

6. Newburger JW, Takahashi M, Gerber MA, Gewitz MH, et al. Diagnosis, treatment, and long-term management of Kawasaki disease: a statement for health professionals from the Committee on Rheumatic Fever, Endocarditis, and Kawasaki Disease, Council on Cardiovascular Disease in the Young, American Heart Association. Pediatrics. 2004; 114(6):1708-33.

7. deZorzi A, ColanSD, Gauvreau K, Baker AL, etal. Coronary artery dimensions may be misclassified as normal in Kawasaki disease. J Pediatr. 1998; 133(2):254-8.

8. McCrindle BW, Rowley AH, Newburger JW, Burns JC, et al. Diagnosis, Treatment, and Long-Term Management of Kawasaki Disease: A Scientific Statement for Health Professionals from the American Heart Association. Circulation. 2017; 135(17):e927-99.

9. Yang HM, Du ZD, Fu PP. Clinical features of recurrent Kawasaki disease and its risk factors. Eur J Pediatr. 2013; 172(12):1641-7.

10. Mortazavi SH, Amin R, Alyasin S, Kashef S, et al. Downregulation of TLR2, 3, 9 and Signaling Mediators, MyD88 and TRIF, Gene Transcript Levels in Patients with Kawasaki Disease Treated with IVIG. Iran J Allergy Asthma Immunol. 2015; 14(2):188-97.

11. Liu MY, Liu HM, Wu CH, Chang CH, et al. Risk factors and implications of progressive coronary dilatation in children with Kawasaki disease. BMC Pediatr. 2017; 17(1):139.

12. Giannouli G, Tzoumaka-Bakoula C, Kopsidas I, Papadogeorgou P, et al. Epidemiology and Risk Factors for Coronary Artery Abnormalities in Children with Complete and Incomplete Kawasaki Disease During a 10-Year Period. Pediatr Cardiol. 2013; 34(6):1476-81.

13. Yoon KL. Update of genetic susceptibility in patients with Kawasaki disease. Korean J Pediatr. 2015; 58(3):84-8.

14. Greco A, De Virgilio A, Rizzo MI, Tombolini M, et al. Kawasaki disease: an evolving paradigm. Autoimmun Rev. 2015; 14(8):703-9.

15. Yeom JS, Woo HO, Park JS, Park ES, et al. Kawasaki disease in infants. Korean J Pediatr. 2013; 56(9):377-82.

16. Baraona F, Valente AM, Porayette P, Pluchinotta FR, Sanders SP. Coronary Arteries in Childhood Heart Disease: Implications for Management of Young Adults. J Clin Exp Cardiolog. 2012; (Suppl 8):006.

17. Galeotti C, Bayry J, Kone-Paut I, Kaveri SV. Kawasaki disease: Aetiopathogenesis and therapeutic utility of intravenous immunoglobulin. Autoimmun Rev. 2010; 9(6):441-8.

18. Kaneko K, Takahashi M, Yoshimura K, Kitao T, et al. Intravenous Immunoglobulin Counteracts Oxidative Stress in Kawasaki Disease. Pediatr Cardiol. 2012; 33(7):1086-8.

19. Qiu H, He Y, Rong X, Ren Y, et al. Delayed intravenous immunoglobulin treatment increased the risk of coronary artery lesions in children with Kawasaki disease at different status. Postgrad Med. 2018; 130(4):442-7.

20. Zhu FH, Ang JY. The clinical diagnosis and management 
of Kawasaki disease: A review and update. Curr Infect Dis Rep. 2016; 18(10):32.

21. Bray C, Bell LN, Liang H, Haykal R, et al. Erythrocyte Sedimentation Rate and C-reactive Protein Measurements and Their Relevance in Clinical Medicine. WMJ. 2016; 115(6):317-21.

22. Chen J, Liu Y, Liu W, Wu Z. A meta-analysis of the biomarkers associated with coronary artery lesions secondary to Kawasaki disease in Chinese children. J Huazhong Univ Sci Technolog Med Sci. 2011; 31(5):705.

23. Gómez de Diego JJ, García Fernández MA, Sales Sales
JR. Enfermedad de Kawasaki estudiada mediante TC multidetectora. Rev Esp Cardiol. 2005; 58(10):1224-5.

24. Goh YG, Ong CC, Tan G, Liang CR, et al. Coronary manifestations of Kawasaki disease in computed tomography coronary angiography. J Cardiovasc Comput Tomogr. 2018; 12(4):275-80.

25. Jrad M, Ben Salem F, Barhoumi C, Lassoued F, et al. The Role of Computed Tomography Coronary Angiography in Kawasaki Disease: Comparison with Transthoracic Echocardiography in a 25-Case RetrospectiveStudy. Pediatr Cardiol. 2019; 40(2):265-75. 